\title{
071
}

\section{FREQUENZA DI ISOLAMENTO DI MICETI PRESSO L'OSPEDALE ONCOLOGICO DI CAGLIARI}

Podda R., Porcu P.P., Sanna M.

Ospedale Oncologico "A.Businco" - Cagliari

Nei reparti di terapia intensiva, talvolta è necessario intervenire con terapie empiriche nei pazienti neutropenici, immunodepressi, portatori di cateteri etc., nei quali si sospetti una infezione micotica o prima che il laboratorio abbia potuto indicare la specie fungina responsabile dell'infezione.

Nella nostra realtà locale, il $40 \%$ delle specie fungine isolate appartiene al genere candida non albicans; considerando che tra queste, alcune come la $C$ krusei $(C K)$ e la $C$. glabrata $(C G)$ sono o possono essere resistenti agli azoli, è stata fatta, un'indagine sulla frequenza delle specie micotiche isolate nel corso dell'anno 2004 suddivise per sito corporeo di provenienza.

\section{Materiali e metodi}

Sono stati analizzati durante lo scorso anno 11.000 campioni. Sono stati seminati su Agar ciccolato, Msa, Mac Conkey e Sabouraud. Le colonie di miceti sono state identificate col sistema Vitek 2 della bioMerieux.

\section{Risultati}

Sono stati isolati 368 ceppi di miceti che risultano distribuiti come indicato nella tabella sottostante

\begin{tabular}{lccccccc}
\hline & CA & CG & CK & CP & SC & CT & altri \\
\hline $\begin{array}{l}\text { Totale } \\
\%\end{array}$ & 223 & 61 & 27 & 20 & 13 & 6 & 18 \\
\hline Basse vie & 53 & 17 & 7 & 5 & 4 & 2 & 5 \\
respirat. & $78 \%$ & $3 \%$ & $12 \%$ & & & $4 \%$ & $3 \%$ \\
\hline Alte vie & 10 & & 3 & & & & 1 \\
respirat. & $71 \%$ & & $21 \%$ & & & $8 \%$ \\
\hline Vie & 131 & 45 & 12 & 10 & 9 & 3 & 14 \\
gen. urin. & $59 \%$ & $20 \%$ & $5 \%$ & $5 \%$ & $4 \%$ & $3 \%$ & $14 \%$ \\
\hline Sist. card. & 5 & & & 8 & & & \\
circolat. & $38 \%$ & & & $32 \%$ & & & \\
\hline Apparat. & 11 & 13 & 3 & & 3 & & 1 \\
digerente & $36 \%$ & $43 \%$ & $10 \%$ & & $10 \%$ & & $1 \%$ \\
\hline Altri & 13 & 1 & 1 & 2 & 1 & & \\
materiali & $73 \%$ & $6 \%$ & $6 \%$ & $9 \%$ & $6 \%$ & & \\
\hline
\end{tabular}

CA: C. albicans CP: C. parapsilosis SC: S. cerevisiae CT: $C$.tropicalis

\section{Conclusioni}

Come si evince dai dati esposti, C. albicans rappresenta il lievito più frequentemente isolato nei campioni positivi provenienti dai distretti delle alte e basse vie respiratorie e dalle vie genito urinarie, mentre nei campioni provenienti dal sistema cardiocircolatorio e dell'apparato digerente prevalgono rispettivamente la $C$. parapsilosis e la $C$. glabrata.

Notevole è anche il numero di ceppi di $C$. krusei isolati nei campioni delle alte e basse vie respiratorie, dall'apparato digerente e di C. glabrata dalle vie genito urinarie. La conoscenza di questi dati che invieremo ai reparti interessati potrà essere d'ausilio nell'intraprendere una accorta terapia empirica in attesa che il laboratorio concluda le indagini e comunichi i risultati definitivi. 